\title{
Systematic Expression Profiling Analysis Identifies Specific MicroRNA-Gene Interactions that May Differentiate between Active and Latent Tuberculosis Infection
}

\author{
Lawrence Shih-Hsin Wu, ${ }^{1}$ Shih-Wei Lee, ${ }^{2,3}$ Kai-Yao Huang, ${ }^{4}$ Tzong-Yi Lee, ${ }^{4,5}$ \\ Paul Wei-Che Hsu, ${ }^{6}$ and Julia Tzu-Ya Weng ${ }^{4,5}$ \\ ${ }^{1}$ Institute of Medical Sciences, Tzu Chi University, Hualien 97004, Taiwan \\ ${ }^{2}$ Taoyuan General Hospital, Ministry of Health and Welfare, Taoyuan 33004, Taiwan \\ ${ }^{3}$ Department of Life Sciences, National Central University, Taoyuan 32001, Taiwan \\ ${ }^{4}$ Department of Computer Science and Engineering, Yuan Ze University, Taoyuan 32003, Taiwan \\ ${ }^{5}$ Innovation Center for Big Data and Digital Convergence, Yuan Ze University, Taoyuan 32003, Taiwan \\ ${ }^{6}$ Bioinformatics Core Laboratory, Institute of Molecular Biology, Academia Sinica, Taipei 11529, Taiwan \\ Correspondence should be addressed to Julia Tzu-Ya Weng; julweng@saturn.yzu.edu.tw
}

Received 24 March 2014; Accepted 1 July 2014; Published 4 September 2014

Academic Editor: Tzu-Hao Chang

Copyright (C) 2014 Lawrence Shih-Hsin Wu et al. This is an open access article distributed under the Creative Commons Attribution License, which permits unrestricted use, distribution, and reproduction in any medium, provided the original work is properly cited.

\begin{abstract}
Tuberculosis (TB) is the second most common cause of death from infectious diseases. About $90 \%$ of those infected are asymptomatic-the so-called latent TB infections (LTBI), with a $10 \%$ lifetime chance of progressing to active TB. To further understand the molecular pathogenesis of TB, several molecular studies have attempted to compare the expression profiles between healthy controls and active TB or LTBI patients. However, the results vary due to diverse genetic backgrounds and study designs and the inherent complexity of the disease process. Thus, developing a sensitive and efficient method for the detection of LTBI is both crucial and challenging. For the present study, we performed a systematic analysis of the gene and microRNA profiles of healthy individuals versus those affected with TB or LTBI. Combined with a series of in silico analysis utilizing publicly available microRNA knowledge bases and published literature data, we have uncovered several microRNA-gene interactions that specifically target both the blood and lungs. Some of these molecular interactions are novel and may serve as potential biomarkers of TB and LTBI, facilitating the development for a more sensitive, efficient, and cost-effective diagnostic assay for TB and LTBI for the Taiwanese population.
\end{abstract}

\section{Introduction}

Tuberculosis (TB) is an infectious disease usually caused by Mycobacterium tuberculosis (Mtb) [1]. Approximately onethird of the world's population is estimated to be latently infected with $M t b$ [2]. In other words, the host immune mechanism can sometimes keep the extent of the bacterial attack arrested at latent TB infection (LTBI). When the host's immune system becomes weakened, LTBI can progress to active pulmonary, or in fewer cases, extrapulmonary TB [3]. In fact, about $90 \%$ of those infected with $M t b$ are asymptomatic, showing signs of LTBI, with a $10 \%$ lifetime chance of developing active TB [4].
In primary active $\mathrm{TB}$, the bacteria overcome the immune system defense and begin to multiply soon after the initial infection [1]. However, in LTBI, the bacteria remain dormant for many years before progressing to active TB. Even after treatment, there is still the risk of reactivation due to immunosuppression, or multiple-drug resistant TB bacteria [5].

Despite the advancement in pulmonary medicine, $\mathrm{TB}$ remains a significant global health issue. The only currently available vaccine is bacillus Calmette-Guérin (BCG), which shows decreased effectiveness after about ten years [5]. The tuberculin skin test (TST) and the interferon-gamma release assays (IGRA) are the usual clinical method for the diagnosis 
of TB and LTBI, with the latter being regarded as the more sensitive assay which measures the amount of interferongamma (IFN- $\gamma$ ) released by blood cells in response to specific $M t b$ antigens [6]. However, these methods often produce false positive results. Thus, developing a sensitive and efficient method for the detection of LTBI and understanding the disease pathology of TB represent a major challenge in the prevention of the disease.

To date, several studies have compared the gene expression profiles between healthy individuals and active TB or LTBI patients [7-10]. These findings reveal important transcriptionally regulated markers of key biological processes, including genes involved in inflammatory responses, immune defense, cell activation, homeostatic processes, and regulation of cell proliferation and apoptosis. It appears that TB and LTBI share similar affected pathways, in which specific molecular markers may be able to discriminate the two disease statuses.

More recent evidence suggests the use of microRNAs as biomarkers for active TB. MicroRNAs (miRNAs) are small, noncoding, single-stranded RNAs that modulate the expression of genes involved in development, cell differentiation, proliferation, and apoptosis [11]. It is estimated that as many as $20 \%$ of all human transcripts are targeted by microRNAs [12]. These tiny RNA molecules, proven to be more stable than messenger RNAs [13], actively circulate in bodily fluids and, thus, are thought to represent a more direct indicator of altered physiology [14]. Indeed, the therapeutic and diagnostic potentials of microRNAs have been the target of extensive study [15], especially in cancer research [16, 17].

In fact, microRNAs have been implicated to play important roles in the disease mechanisms of various infectious diseases. For example, the mouse microRNA, mmu-miR29 , has been shown to target IFN- $\gamma$ and suppress immune responses against intracellular pathogens [18]. The human microRNA hsa-miR-32 has been found to modulate retrovirus PFV-1 replication [19]. Moreover, specific microRNAgene interactions appeared to regulate the pathogenesis of HIV-1 infection [20].

Recently, through the use of expression array technology to explore the transcriptome on a global scale, several groups have investigated the possibility of using microRNAs or specific microRNA-gene associations as biomarkers for the diagnosis of TB or the differentiation between active TB and LTBI. These gene and microRNA expression studies identified candidate genes and microRNAs involved in cytokine and chemokine responses, inflammation, and intracellular trafficking in the progression from latent infection to active TB [7, 21-24]. Unfortunately, few of these findings are consistent with each other. Many of the discovered molecular markers vary due to diverse genetic background of the study population, differences in the study design, and the inherent complexity of the disease process.

With the latest advances in technology and bioinformatics, we believe utilizing complementary platforms to examine the differences in transcriptome between TB and LTBI in the Taiwanese population will help uncover novel biomarkers and build upon the knowledge regarding the disease diagnosis and pathology. Here, we present a systematic approach of combining gene and microRNA expression profiling to uncover the complex networks of molecular interactions associated with TB and LTBI. Our study began with the analysis of gene and microRNA expression profiles among active TB, LTBI, and healthy individuals. Candidate genes and microRNAs that appeared to be inversely correlated in expression were subsequently selected for a series of bioinformatics analyses to determine the nature of their relationships. Based on the computational predictions, a comprehensive microRNA-gene interaction network was constructed, revealing previously validated and novel molecular signatures that help improve our understanding and the diagnostic differentiation of TB and LTBI.

\section{Materials and Methods}

The analytical flow of the present study is illustrated in Figure 1. Profiling of microRNA and gene expression was performed to identify differentially expressed transcripts among 7 healthy control, 7 active TB, and 7 LTBI individuals. Differentially expressed candidates were categorized into upand downregulated genes and microRNAs, and divided into three groups: TB versus healthy control, LTBI versus healthy control, and LTBI versus TB. Putative microRNAs targeting the differentially expressed transcripts were predicted using six microRNA knowledge bases.

2.1. Clinical Sample Collection. All procedures were reviewed and approved by the Institutional Review Board of Taoyuan General Hospital, Ministry of Health and Welfare, Taoyuan, Taiwan. Written informed consents were obtained from all participants. Eligibility for entry into the study was based on clinical signs and symptoms of $M t b$ infection. LTBI subjects were recruited from close contact with active TB patients, with positive T-SPOT TB test and negative chest radiograph, but without clinical evidence of active TB. Healthy controls were individuals who had not been in close contact with TB or LTBI patients and showed no clinical signs of TB or LTBI. Individuals with allergic diseases, diabetes, cancer, immunecompromised conditions, and coinfections with any types of infectious diseases were excluded. In total, seven healthy individuals, seven patients with active $\mathrm{TB}$, and seven subjects with LTBI were included in the present study.

2.2. RNA Isolation. RNA was isolated from peripheral blood mononuclear cells. RNA quality was determined by an optical density (OD) 260/280 ratio $\geq 1.8$ and OD 260/230 ratio $\geq$ 1.5 on a spectrophotometer and by the intensity of the $18 \mathrm{~S}$ and $28 \mathrm{~S}$ rRNA bands on a $1 \%$ formaldehyde-agarose gel. RNA quantity was detected by a spectrophotometer. RNA integrity was examined on an Agilent Bioanalyzer. RNA with a RNA integrity number (RIN) $\geq 6.0$ and $28 \mathrm{~S} / 18 \mathrm{~S}>0.7$ was subjected to microarray analysis.

2.3. MicroRNA and Gene Expression Analysis. RNA samples were subjected to Human OneArray v6 and Human microRNA OneArray v5 (Phalanx Biotech, Hsinchu, Taiwan). Data were analyzed with Rosetta Resolver System software (Rosetta Biosoftware, USA). Standard selection criteria 


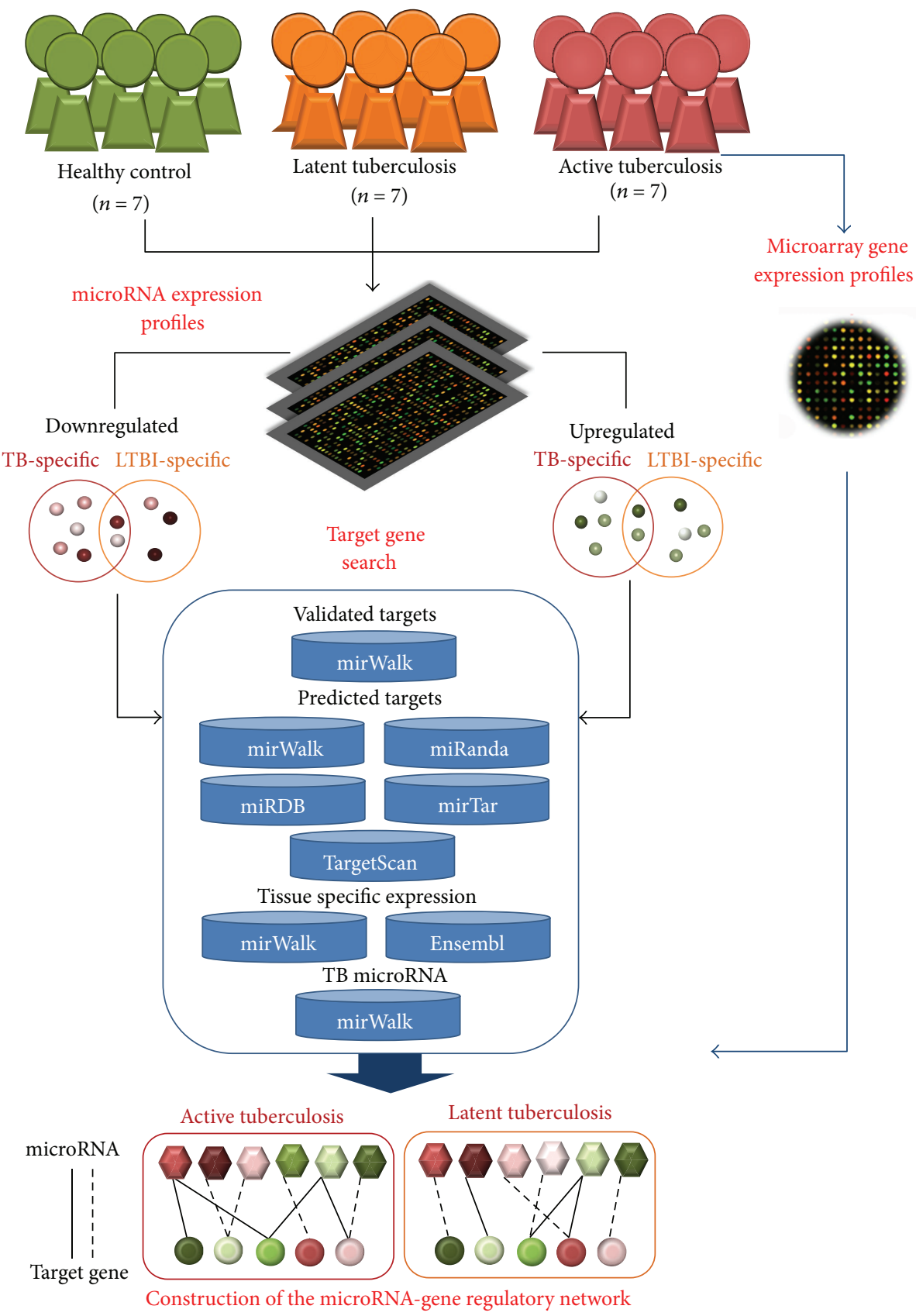

Figure 1: System flow of our analysis.

to identify differentially expressed genes were (1) absolute $\log _{2}$ fold change $\geq 1$; (2) false discovery rate of $<0.05$; (3) the intensity difference between two samples under comparison $\geq 1000$; (4) individual intensity $\geq 500$. Genes and microRNAs showing significant differential expression were categorized into TB versus healthy control, LTBI versus healthy control, and LTBI versus TB.

2.4. Bioinformatics Analysis. The candidate microRNAs were analyzed for associations with the candidate genes to reveal potential microRNA-gene interactions, in which decreased microRNA expression may be correlated with increased target gene expression and vice versa. In order to establish an in silico correlation between the microRNA profile in the blood and lung tissues, we input our list of differentially expressed microRNAs on miRWalk [25], a database that curates published results on experimentally verified microRNA tissue specificity, target genes, and disease associations. Next, for the selected microRNA candidates that have been shown to be active in both blood and lungs, a list of validated target genes was obtained from miRWalk [25]. For microRNAs with no validated target genes, target gene predictions were obtained by comparing among six microRNA knowledge bases, including miRWalk [25], miRTar [26], miRDB [27], miRANDA [28], RNA22 [29], and TargetScan [30], so as to take advantage of the strengths of each microRNA target 
TABLE 1: Number of differentially expressed genes and microRNAs among TB, LTBI, and healthy controls.

\begin{tabular}{lcc}
\hline Comparison & Upregulated & Downregulated \\
\hline Genes & & \\
TB versus control & 16 & 111 \\
LTBI versus control & 31 & 267 \\
LTBI versus TB & 105 & 67 \\
mircroRNA & & \\
TB versus control & 0 & 1 \\
LTBI versus control & 16 & 6 \\
LTBI versus TB & 9 & 1 \\
\hline
\end{tabular}

Absolute fold change $\geq 1$, FDR $<0.05$.

prediction algorithm. A gene would be included in the target gene list if four out of these six databases generated the same prediction. The validated and predicted genes were then compared with differentially expressed microRNA-induced upregulated and downregulated genes in TB and LTBI. For matched genes, their tissues-specific expression profiles were determined through a search on the Ensembl system [31]. Only those target genes expressed in both blood and lungs were selected. Candidate microRNAs that have been implicated in TB were also determined through a search with the disease target tool in miRWalk [25].

2.5. MicroRNA-Gene Interaction Analysis. STRING (v9.1) $[32,33]$ was utilized to determine any known and putative interactions among the differentially expressed genes that were also estimated to be targeted by the differentially expressed microRNAs. The predicted relationships were based on a confidence level of 0.7 , coexpression evidence, experimental validation, or database detection. To visualize the relationships among these TB- and LTBI-specific molecular signatures, a potential interaction network incorporating expression information was built with Cytoscape v3.1.0 [34]. In addition, genes targeted by TB-related microRNAs were mapped to KEGG pathways [35] by the gene set enrichment function in miRTar [26].

\section{Results}

Between LTBI and TB, 172 genes and 10 microRNAs presented significant differences in expression (Table 1). Additionally, there were 11 upregulated and 111 downregulated genes, plus one downregulated microRNA between TB and healthy controls. In contrast, 31 genes and 16 microRNAs showed increased expression, while 267 genes as well as six microRNAs exhibited decreased expression in LTBI compared to healthy individuals.

By using the validated target organ search in miRWalk [25], 11 out of the 30 differentially expressed microRNAs were found to target both blood and lungs. We hypothesize that these microRNAs represent potential correlates between the molecular profiles between the two tissues. Therefore, subsequent analyses were focused on these candidate microRNAs.

To uncover the potential microRNA-mediated regulation underlying the gene expression differences between $\mathrm{TB}$ and
LTB, we utilized six microRNA target prediction databases and cross-validated the results to find putative microRNAgene interactions (Table 2). Disease target search in miRWalk [25] indicated that some of these microRNAs have been implicated in other TB studies. However, our study also identified some novel microRNA-gene interactions that exhibited differential expression among TB, LTBI, and healthy controls.

The STRING [32, 33] network mapping tool was also employed to identify coexpressing or experimentally validated relationships among the candidate genes. The systematic prediction results based on validated and predicted gene, tissue, and disease targets were used to link the microRNAs and target genes in an interaction network (Figure 2). TBand LTBI-specific differentially expressed microRNAs and genes may be involved in complex and dynamic interactions. The integration of the differential expression patterns in this graphical representation revealed the potential regulatory relationships among these molecular signatures.

Finally, by performing a disease target search, we identified candidate microRNAs that have also been associated with TB in the literature (Table 2). These TB-related microRNAs may be important key molecules specific to the disease pathology. In particular, the association between hsa-miR$150-5 \mathrm{p}$ and $\beta$-arrestin 2 (ARRB2) gene was mapped to the chemokine signaling pathway. Interestingly, LTBI-specific upregulated hsa-miR-16-5p and TB-specific upregulated hsalet-7i-5p both target the same pathogenic infection pathway, though via different genes (Figure 3 ). In addition, microRNA hsa-miR-221-3p, with its target FOS (FBJ murine osteosarcoma viral oncogene homolog), were predicted to be involved in MAPK, B-cell receptor, and T-cell receptor signaling pathways.

\section{Discussion}

In this study, we integrated the available gene and microRNA expression array technology and bioinformatics tools to investigate the possibility of uncovering molecular events indicative of TB and LTBI. Our system flow allowed us to assess the results of gene and microRNA expression profiling through a combination of computational prediction and validation with published data.

Some of the candidate microRNAs identified by other groups were confirmed in our analysis, though with some inconsistencies in expression pattern. For example, microRNA hsa-miR-146a-5p appeared to be downregulated in TB patients in Spinelli et al.s study [36] but was found to be upregulated by Furci et al. [37]. As well, microRNA hsa-miR-223-3p showed reduced expression in TB in one study [24] but enhanced expression in another published finding [38]. Our analysis, however, revealed downregulated hsa-miR-223-3p expression specific to LTBI. Whereas Meng et al. observed a lower expression of hsa-miR-150-5p in LTBI compared with healthy controls [39], we found the microRNA being expressed at a higher level in LTBI relative to TB. On the other hand, consistent with $[22,40]$, we found the level of hsa-miR-142-3p and hsa-miR-21-5p expression to be enhanced in LTBI relative to TB, and hsa-let-7i-5p expression, increased in TB compared with healthy controls. 
TABLE 2: Selected blood- and lung-targeting microRNAs and their validated or predicted target genes.

\begin{tabular}{|c|c|}
\hline microRNA & Target genes \\
\hline \multicolumn{2}{|c|}{ TB versus control downregulated } \\
\hline hsa-let-7i-5p & ADM, HMGA2, LRRK2, SERPING1, TLR4 \\
\hline \multicolumn{2}{|c|}{ LTBI versus control upregulated } \\
\hline hsa-miR-107 & BTG2, EIF5 \\
\hline hsa-miR-142-3p $\mathrm{p}^{\mathrm{a}, \mathrm{b}}$ & KLF4, POMC, JUN, PMAIP1, PDGFRB, SIK1 \\
\hline hsa-miR-16-5p & SIK1, NR4A1, IER2, AGPAT4, CSRNP1, PLEKHG2, CD69 \\
\hline hsa-miR-21-5p $p^{b}$ & CD69, H3F3B, TAF1C, TRIB1, PMAIP1 \\
\hline hsa-miR-22-3p & H3F3B, TAF1C, TRIB1, PMAIP1, BTG2 \\
\hline hsa-miR-221-3p & FOS, ZBTB24, EIF1, MYLIP, NAP1L5, SOCS3 \\
\hline hsa-miR-223-3p ${ }^{b}$ & RHOB, CXCL2 \\
\hline \multicolumn{2}{|c|}{ LTBI versus TB upregulated } \\
\hline hsa-miR-146a-5p ${ }^{b}$ & ST20 \\
\hline hsa-miR-150-5p ${ }^{b}$ & CPD, ARRB2, FFAR2, NUP214, PNMA3, C20orf24, C16orf57 \\
\hline hsa-miR-16-5p & CPD, C15orf39, C16orf57, TUBA1A \\
\hline hsa-miR-221-3p & ANXA1, FOS, PLAUR, TIMP2, C16orf57, MIDN \\
\hline
\end{tabular}

The discrepancies among studies emphasize the influence of genetic background and experimental design on the study results, underscoring the difficulty of deciphering the molecular mechanisms underlying TB pathology.

Yet, the most interesting inconsistency between our results and other studies is probably the expression pattern of hsa-miR-223 among active TB, LTBI, and healthy subjects. While Wang et al. [23] observed enhanced expression of hsa-miR-223 in TB patients versus nonactive TB group, the same microRNA appeared to be most abundant in healthy individuals, though the latter group was able to successfully validate their observation in a murine model. We, on the other hand, found this microRNA to be expressed at a higher level in individuals latently infected with TB.

Nevertheless, similar to Dorhoi et al's results [38], our computational prediction, coupled with the gene expression array data, showed an inverse correlation in expression patterns between hsa-miR-223 and its target gene CXCL2, a chemokine, that is, synthesized to facilitate an inflammatory response after injuries [41]. Deletion of miR-223 resulted in increased susceptibility to TB infection in mice and significantly augmented CXCL2 production, but upon neutralization of CXCL2, the severity of TB infection could be slightly reversed [38]. Thus, it appears that hsa-miR-223, as well as its modulation of CXCL2 abundance, may be able to determine, at least in part, the chance that an individual would succumb to a full-blown TB infection. This makes sense in the context of our finding, in which LTBI individuals possessed a higher level of hsa-miR-223 expression. It is likely that hsa-miR-223 transcription is induced upon the initial $M t b$ attack to arrest the infection at a latent state in individuals with LTBI.

In addition, we identified a potential interaction between hsa-miR-150-5p and the ARBB2 ( $\beta$-arrestin 2) gene, an immune regulator involved in cell adhesion, migration, and proliferation [42]. In particular, ARBB2 modulates the activity of $\mathrm{G}$ protein-coupled receptors to facilitate downstream inflammatory and immune responses [42]. The role that ARBB2 plays in the immune system is evidenced by its association with toll-like receptors, cytokines, chemokines, and various cell cycle and cell stress regulating signaling pathways in diseases such as rheumatoid arthritis, endotoxemia, sepsis, asthma, multiple sclerosis, and atherosclerosis [43]. Moreover, differential expression of $A R B B 2$ has been observed in tuberculosis $[44,45]$ and reduced expression of $A R B B 2$ has been correlated with an augmented level of IFN- $\gamma$ [46], underscoring the importance of ARBB2 in the regulation of immune response. In our study, LTBIspecific downregulated $A R B B 2$ gene is the predicted target of the LTBI-specific upregulated hsa-miR-150-5p. The inverse relationship may indicate that this particular interaction plays a regulatory role in the immune response against $M t b$ bacteria.

Furthermore, we have also uncovered novel microRNAgene interactions that may regulate the disease progression from latent to active TB. For example, in LTBI, both hsamir-16-5p and hsa-mir-221-3p appeared to be significantly upregulated compared with $\mathrm{TB}$ and healthy control. The microRNA hsa-mir-16 is a known inducer of apoptosis [47]. Our miRNA-gene interaction network revealed TUBA1A (tubulin alpha-1A) as a novel target gene that may modulate the host response to pathogenic infections through the interaction with hsa-mir-16-5p. Interestingly, TB-specific downregulated microRNA hsa-let-7i and its target gene TLR4, a toll-like receptor involved in innate immunity [48], were also mapped to the same pathway. Moreover, the potential interaction between hsa-miR-221-3p and FOS was linked to the $\mathrm{T}$-cell and $\mathrm{B}$-cell receptor signaling pathways. The FOS gene regulates various important biological processes such as cell proliferation, differentiation, and survival [49]. The potential interaction between the differentially expressed microRNAs and their target genes plays important roles in 
LTBI versus HC

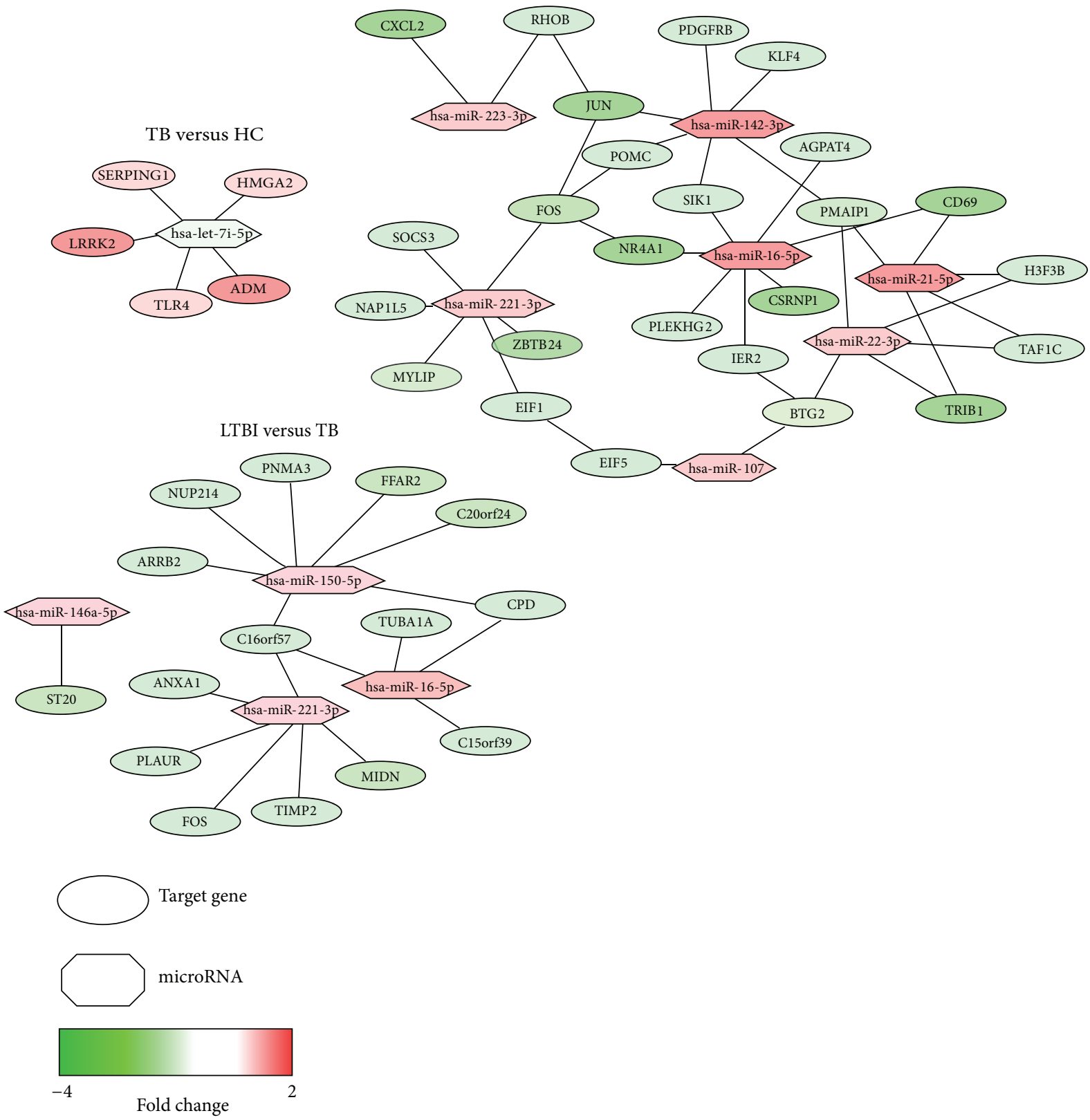

FIgURE 2: Potential TB- and LTBI-differentiating microRNA-gene interaction network. Color intensity indicates the expression of each molecule. Red indicates upregulated expression and green indicates downregulated expression.

innate and adaptive immune responses in TB pathology. They may be used as molecular identifiers indicative of the two infection states.

In conclusion, we have performed a complementary analysis of gene and microRNA expression profiling and established a comprehensive microRNA-gene interaction network that may help differentiate between TB and LTBI. Note that our work is limited by the small sample size, and therefore the biomarkers may be specific to this particular study group. However, the strength of our analysis lies in the integration of various publicly available computational tools and experimental resources, allowing us to identify the most promising microRNA-gene associations through in silico predictions, gene expression profiling data, and published findings. Further, in hope to identify microRNAgene interactions in the blood that would represent specific physiological conditions in the lungs, we filtered the candidate microRNAs and the corresponding target genes based on the similarity of their regulatory relationships in these two tissues.

Our work built on the emerging evidence that microRNA-gene interactions can be used as useful clinical 


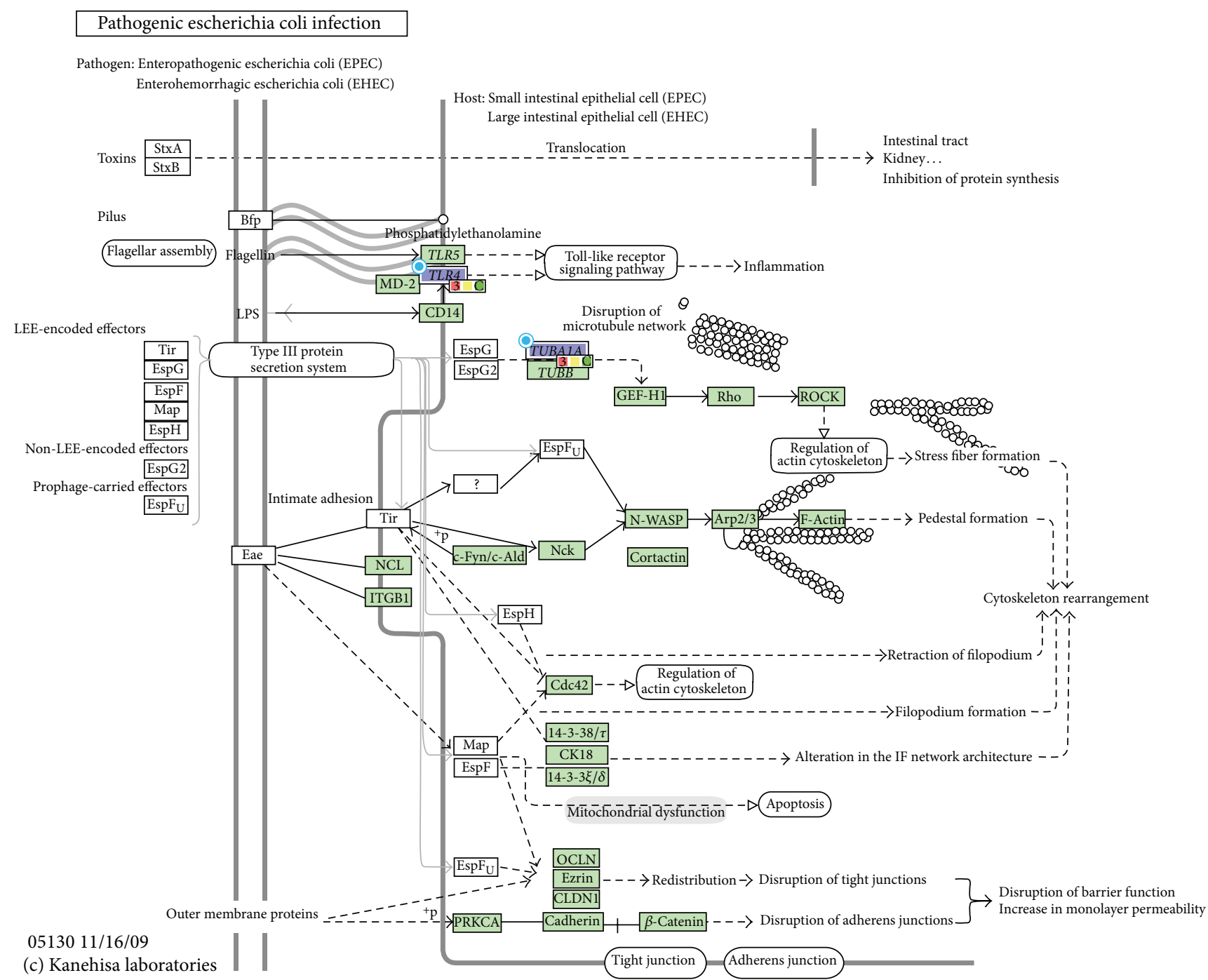

FIGURE 3: Interactions between hsa-miR-16-5p and TUBA1A, and hsa-let-7i-5p and TLR4 that are predicted to be involved in the host response against pathogen infection.

biomarkers. Moreover, we have also uncovered novel TB and LTBI biomarkers specific to the Taiwanese population. These new molecular signatures are based on microRNA-gene interactions that may reflect the differences in TB disease state, further our understanding of TB pathogenesis, and facilitate the development of a molecular diagnostic platform for LTBI detection.

\section{Conflict of Interests}

The authors declare that there is no conflict of interests regarding the publication of this paper.

\section{Authors' Contribution}

Lawrence Shih-Hsin Wu, Shih-Wei Lee, and Julia Tzu-Ya Weng conceived and designed the experiments. Kai-Yao Huang, Tzong-Yi Lee, Paul Wei-Che Hsu, and Julia TzuYa Weng analyzed the data. Julia Tzu-Ya Weng wrote the paper. Lawrence Shih-Hsin Wu and Shih-Wei Lee contributed equally to this work.

\section{Acknowledgments}

This study was supported by the Ministry of Health and Welfare and Taoyuan General Hospital, Taiwan, under contracts PTH10227 and PTH10207, respectively, as well as the National Science Council of the Republic of China under Contract nos. NSC 101-2628-E-155-002-MY2 and NSC102-2221-E-155-069.

\section{References}

[1] V. Kumar and S. L. Robbins, Robbins Basic Pathology, Saunders/Elsevier, 8th edition, 2007.

[2] C. Dye and B. G. Williams, "The population dynamics and control of tuberculosis," Science, vol. 328, no. 5980, pp. 856-861, 2010. 
[3] I. Smith, "Mycobacterium tuberculosis pathogenesis and molecular determinants of virulence," Clinical Microbiology Reviews, vol. 16, no. 3, pp. 463-496, 2003.

[4] R. L. Skolnik, Global health 101, Jones \& Barlett Learning, 2nd edition, 2012

[5] S. D. Lawn and A. I. Zumla, "Tuberculosis," The Lancet, vol. 378, no. 9785 , pp. 57-72, 2011.

[6] V. Herrera, S. Perry, J. Parsonnet, and N. Banaei, "Clinical application and limitations of interferon- $\gamma$ release assays for the diagnosis of latent tuberculosis infection," Clinical Infectious Diseases, vol. 52, no. 8, pp. 1031-1037, 2011.

[7] M. Jacobsen, D. Repsilber, A. Gutschmidt et al., "Candidate biomarkers for discrimination between infection and disease caused by Mycobacterium tuberculosis," Journal of Molecular Medicine, vol. 85, no. 6, pp. 613-621, 2007.

[8] P. Constantoulakis, E. Filiou, N. Rovina et al., "In vivo expression of innate immunity markers in patients with mycobacterium tuberculosis infection," BMC Infectious Diseases, vol. 10, article 243, 2010.

[9] L. M. Verhagen, A. Zomer, M. Maes et al., "A predictive signature gene set for discriminating active from latent tuberculosis in Warao Amerindian children," BMC Genomics, vol. 14, no. 1, article 74, 2013.

[10] J. Maertzdorf, D. Repsilber, S. K. Parida et al., "Human gene expression profiles of susceptibility and resistance in tuberculosis," Genes and Immunity, vol. 12, no. 1, pp. 15-22, 2011.

[11] L. He and G. J. Hannon, "MicroRNAs: small RNAs with a big role in gene regulation," Nature Reviews Genetics, vol. 5, no. 7, pp. 522-531, 2004.

[12] R. J. Perera and A. Ray, "MicroRNAs in the search for understanding human diseases," BioDrugs, vol. 21, no. 2, pp. 97-104, 2007.

[13] A. Liu, M. T. Tetzlaff, P. VanBelle et al., "MicroRNA expression profiling outperforms mRNA expression profiling in formalinfixed paraffin-embedded tissues," International Journal of Clinical and Experimental Pathology, vol. 2, no. 6, pp. 519-527, 2009.

[14] S. Gilad, E. Meiri, Y. Yogev et al., "Serum microRNAs are promising novel biomarkers," PLoS ONE, vol. 3, no. 9, Article ID e3148, 2008.

[15] A. Jackson and P. S. Linsley, "The therapeutic potential of microRNA modulation," Discovery Medicine, vol. 9, no. 47, pp. 311-318, 2010.

[16] G. A. Calin and C. M. Croce, "MicroRNA signatures in human cancers," Nature Reviews Cancer, vol. 6, no. 11, pp. 857-866, 2006.

[17] M. Ferracin, A. Veronese, and M. Negrini, "Micromarkers: MiRNAs in cancer diagnosis and prognosis," Expert Review of Molecular Diagnostics, vol. 10, no. 3, pp. 297-308, 2010.

[18] F. Ma, S. Xu, X. Liu et al., “The microRNA miR-29 controls innate and adaptive immune responses to intracellular bacterial infection by targeting interferon- $\gamma$," Nature Immunology, vol. 12 , no. 9, pp. 861-869, 2011.

[19] C. H. Lecellier, P. Dunoyer, K. Arar et al., "A cellular microRNA mediates antiviral defense in human cells," Science, vol. 308, no. 5721, pp. 557-560, 2005.

[20] K. Duskova, P. Nagilla, H.-S. Le et al., "MicroRNA regulation and its effects on cellular transcriptome in Human Immunodeficiency Virus-1 (HIV-1) infected individuals with distinct viral load and CD4 cell counts," BMC Infectious Diseases, vol. 13, no. 1, article 250, 2013.
[21] C. Y. Lu, J. Wu, H. H. Wang et al., "Novel biomarkers distinguishing active tuberculosis from latent infection identified by gene expression profile of peripheral blood mononuclear cells," PLoS ONE, vol. 6, no. 8, Article ID e24290, 2011.

[22] Y. Fu, Z. Yi, X. Wu, J. Li, and F. Xu, "Circulating microRNAs in patients with active pulmonary tuberculosis," Journal of Clinical Microbiology, vol. 49, no. 12, pp. 4246-4251, 2011.

[23] C. Wang, S. Yang, G. Sun et al., "Comparative mirna expression profiles in individuals with latent and active tuberculosis," PLoS ONE, vol. 6, no. 10, Article ID e25832, 2011

[24] M. P. R. Berry, C. M. Graham, F. W. McNab et al., "An interferoninducible neutrophil-driven blood transcriptional signature in human tuberculosis," Nature, vol. 466, no. 7309, pp. 973-977, 2010.

[25] H. Dweep, C. Sticht, P. Pandey, and N. Gretz, "MiRWalkdatabase: prediction of possible miRNA binding sites by "walking" the genes of three genomes," Journal of Biomedical Informatics, vol. 44, no. 5, pp. 839-847, 2011.

[26] J. B. Hsu, C.-M. Chiu, S.-D. Hsu et al., "MiRTar: an integrated system for identifying miRNA-target interactions in human," BMC Bioinformatics, vol. 12, article 300, 2011.

[27] X. Wang, "miRDB: a microRNA target prediction and functional annotation database with a wiki interface," $R N A$, vol. 14, no. 6, pp. 1012-1017, 2008.

[28] B. John, A. J. Enright, A. Aravin, T. Tuschl, C. Sander, and D. S. Marks, "Human microRNA targets," PLoS Biology, vol. 2, no. 11, article e363, 2004.

[29] P. Loher and I. Rigoutsos, "Interactive exploration of RNA22 microRNA target predictions," Bioinformatics, vol. 28, no. 24, pp. 3322-3323, 2012.

[30] B. P. Lewis, C. B. Burge, and D. P. Bartel, "Conserved seed pairing, often flanked by adenosines, indicates that thousands of human genes are microRNA targets," Cell, vol. 120, no. 1, pp. 15-20, 2005.

[31] P. Flicek, M. R. Amode, D. Barrell et al., "Ensembl 2014," Nucleic Acids Research, vol. 42, pp. D749-D755, 2014.

[32] D. Szklarczyk, A. Franceschini, M. Kuhn et al., "The STRING database in 2011: functional interaction networks of proteins, globally integrated and scored," Nucleic Acids Research, vol. 39, no. 1, pp. D561-D568, 2011.

[33] A. Franceschini, D. Szklarczyk, S. Frankild et al., "STRING v9.1: protein-protein interaction networks, with increased coverage and integration," Nucleic Acids Research, vol. 41, no. 1, pp. D808D815, 2013.

[34] P. Shannon, A. Markiel, O. Ozier et al., "Cytoscape: a software environment for integrated models of biomolecular interaction networks," Genome Research, vol. 13, no. 11, pp. 2498-2504, 2003.

[35] M. Kanehisa and S. Goto, "KEGG: kyoto encyclopedia of genes and genomes," Nucleic Acids Research, vol. 28, no. 1, pp. 27-30, 2000.

[36] S. V. Spinelli, A. Diaz, L. D’Attilio et al., "Altered microRNA expression levels in mononuclear cells of patients with pulmonary and pleural tuberculosis and their relation with components of the immune response," Molecular Immunology, vol. 53, no. 3, pp. 265-269, 2013.

[37] L. Furci, E. Schena, P. Miotto, and D. M. Cirillo, "Alteration of human macrophages microRNA expression profile upon infection with Mycobacterium tuberculosis," International Journal of Mycobacteriology, vol. 2, no. 3, pp. 128-134, 2013. 
[38] A. Dorhoi, M. Iannaccone, M. Farinacci, K. C. Fae, J. Schreiber, and P. Moura-Alves, "MicroRNA-223 controls susceptibility to tuberculosis by regulating lung neutrophil recruitment," Journal of Clinical Investigation, vol. 123, pp. 4836-4848, 2013.

[39] Q.-L. Meng, F. Liu, X.-Y. Yang et al., "Identification of latent tuberculosis infection-related microRNAs in human U937 macrophages expressing Mycobacterium tuberculosis Hsp16.3," BMC Microbiology, vol. 14, article 37, 2014.

[40] K. Kleinsteuber, K. Heesch, S. Schattling et al., "Decreased expression of miR-21, miR-26a, miR-29a, and miR-142-3p in CD4(+) $\mathrm{T}$ cells and peripheral blood from tuberculosis patients," PLoS ONE, vol. 8, no. 4, Article ID e61609, 2013.

[41] D. A. Armstrong, J. A. Major, A. Chudyk, and T. A. Hamilton, "Neutrophil chemoattractant genes KC and MIP-2 are expressed in different cell populations at sites of surgical injury," Journal of Leukocyte Biology, vol. 75, no. 4, pp. 641-648, 2004.

[42] D. Jiang, T. Xie, J. Liang, and P. W. Noble, "beta-Arrestins in the immune system," Progress in Molecular Biology and Translational Science, vol. 118, pp. 359-393, 2013.

[43] K. Loniewski, Y. Shi, J. Pestka, and N. Parameswaran, “Toll-like receptors differentially regulate GPCR kinases and arrestins in primary macrophages," Molecular Immunology, vol. 45, no. 8, pp. 2312-2322, 2008.

[44] G. C. K. W. Koh, M. F. Schreiber, R. Bautista et al., "Host responses to melioidosis and tuberculosis are both dominated by interferon-mediated signaling," PLoS ONE, vol. 8, no. 1, Article ID e54961, 2013.

[45] S. Mehra, B. Pahar, N. K. Dutta et al., "Transcriptional reprogramming in nonhuman primate (Rhesus Macaque) tuberculosis granulomas," PLoS ONE, vol. 5, no. 8, Article ID el2266, 2010.

[46] R. Xia, Z. Hu, Y. Sun et al., "Overexpression of $\beta$-arrestin 2 in peripheral blood mononuclear cells of patients with cryptococcal meningitis," Journal of Interferon and Cytokine Research, vol. 30, no. 3, pp. 155-161, 2010.

[47] J. Shen, R. Wan, G. Hua et al., "miR-15b and miR-16 induce the apoptosis of rat activated pancreatic stellate cells by targeting Bcl-2 in vitro," Pancreatology, vol. 12, no. 2, pp. 91-99, 2012.

[48] R. Medzhitov, P. Preston-Hurlburt, and C. A. Janeway Jr., "A human homologue of the Drosophila toll protein signals activation of adaptive immunity," Nature, vol. 388, no. 6640, pp. 394-397, 1997.

[49] K. Milde-Langosch, "The Fos family of transcription factors and their role in tumourigenesis," European Journal of Cancer, vol. 41, no. 16, pp. 2449-2461, 2005. 

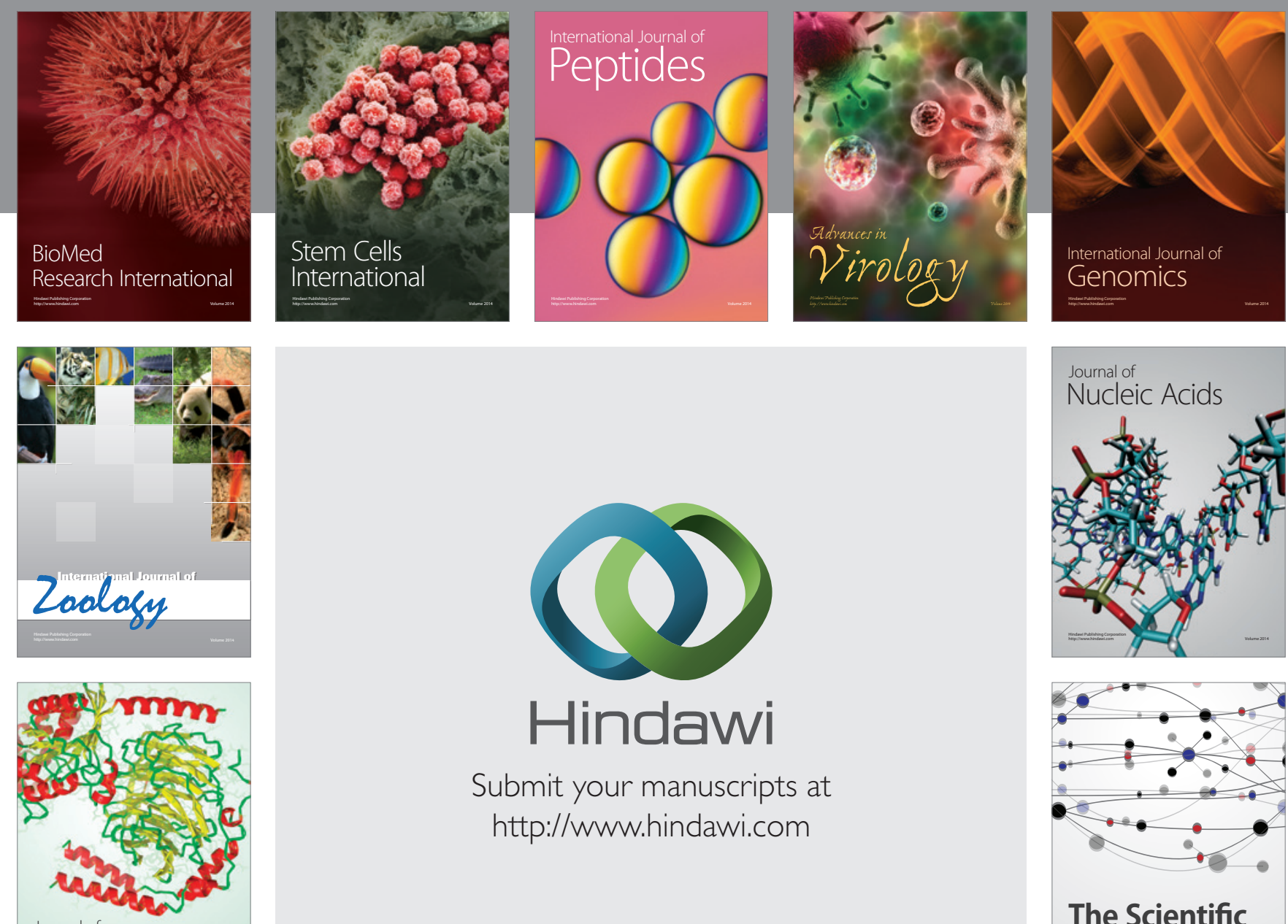

Submit your manuscripts at

http://www.hindawi.com

Journal of
Signal Transduction
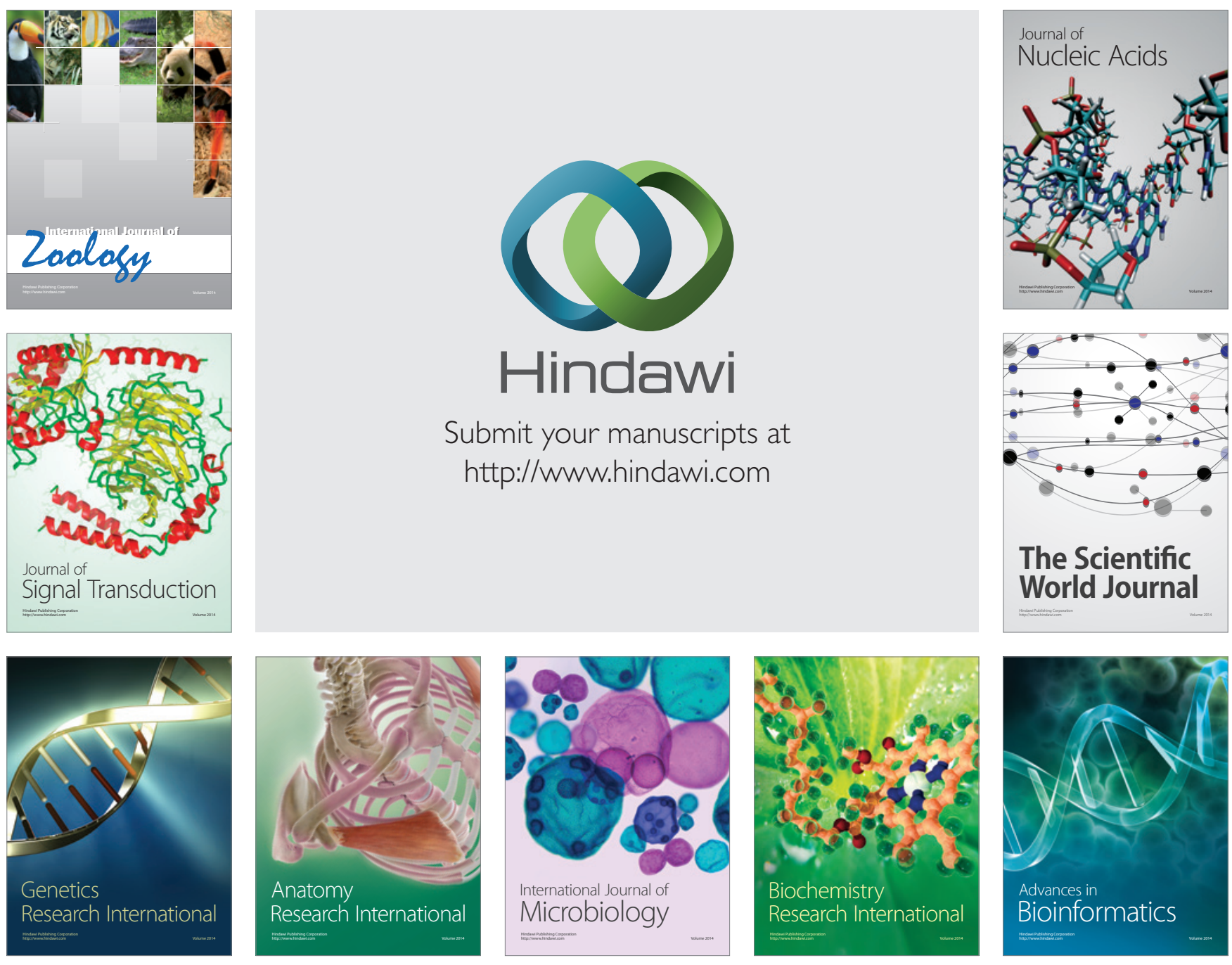

The Scientific World Journal
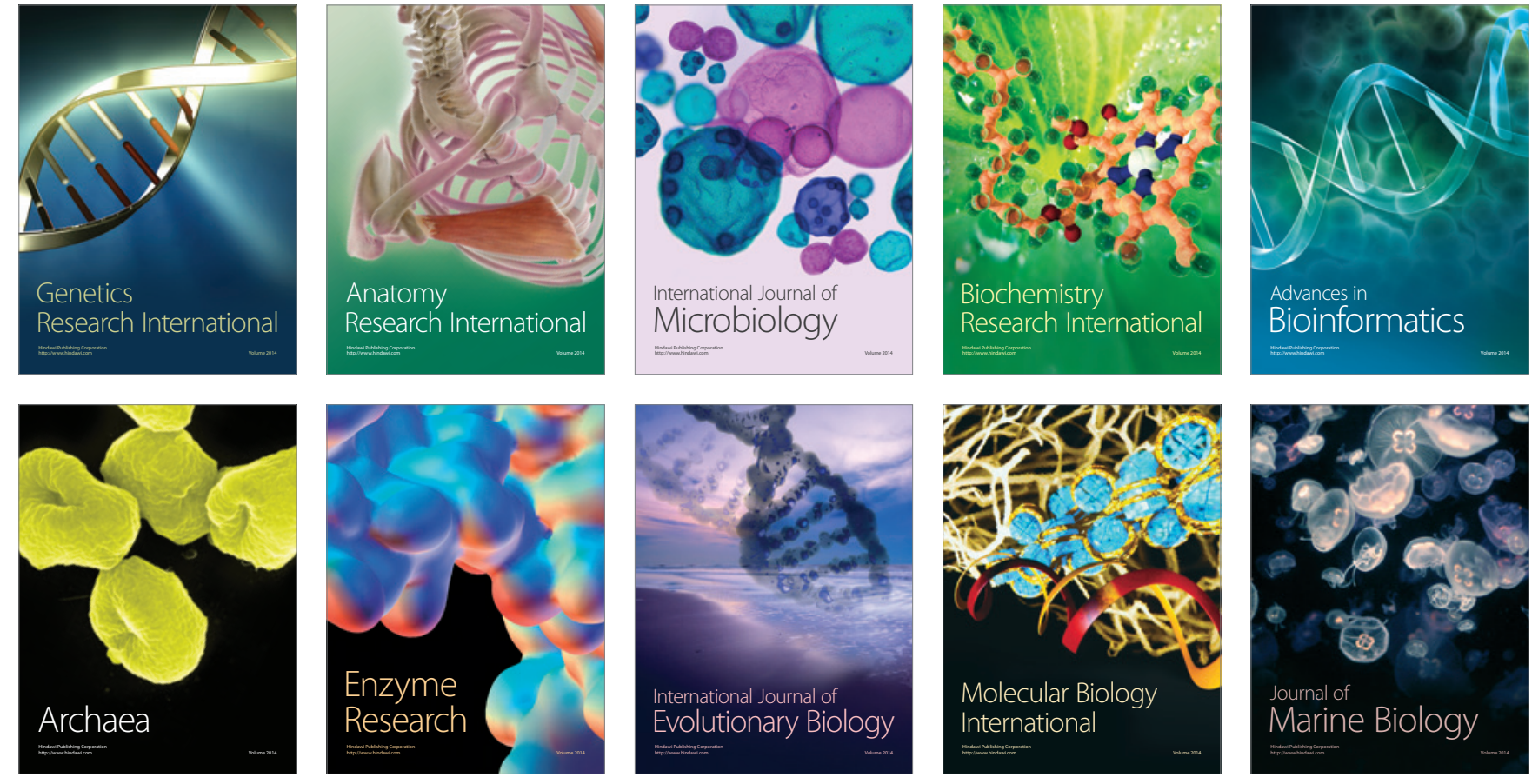